\title{
Epidemiological Studies of Potent Environmental Pathogen: Streptococcus pneumoniae
}

\author{
Nazir A. Brohi", Agha A. Noor, Shaista B. Memon and Sarfraz A. Tunio \\ Institute of Microbiology, University of Sindh, Jamshoro Sindh, Pakistan-76080. \\ *Corresponding Author Email: ahmednazeerbrohi@gmail.com \\ Received 10 May 2016, Revised 07 November 2016, Accepted 09 November 2016
}

\begin{abstract}
A general survey for six months was undertaken for the prevalence of environmental bacterium Streptococcus pneumoniae among the different age groups (3-65 years) including both sexes from various hospitals of Hyderabad city. Laboratory examinations revealed $S$. pneumoniae as most potent environmental pathogen from the sputum and throat swabs of old aged patients and children respectively. During observations, 39 specimens were growth positive; the biochemistry of isolates revealed that they were coagulase, catalase and oxidase negative, TSI, gel hydrolysis positive and were able to ferment glucose, lactose, maltose, galactose, fructose, sucrose, starch and raffinose. The results of antimicrobial activity showed that pneumococci were resistant to the cefspan, septran, cravit, pipemetic acid, azomax, bacitracin, and penicillin and a clear zone of inhibition was observed on clithromycin, optochin, cefizox, genatamycin, minocyclin, levoflaxacin, and vancomycin. There were intermediate zone of inhibition found on claforan, nalidixic acid, amoxycillin, fosfomycin, fortum, and erythromycin on Mueller Hinton's agar after 24 hours incubation.
\end{abstract}

Keywords: Streptococcus penumoniae, Environmental pathogens, Laboratory media, Throat swab, Sputum, Antibiotic sensitivity pattern.

\section{Introduction}

The environment play an important role in transmission of microbial agents to humans, with many environmental materials serving as vehicles Contamination of various objects by potential pathogenic microorganisms is of public health importance as contaminated materials can be possible sources of transmission of such pathogens. Environmental pathogens are the microorganisms that normally spend a substantial part of their lifecycle outside human hosts, but when introduced to humans cause disease with measurable frequency. These pathogens include pathogenic bacteria, protozoa, worms, viruses, prions and fungi. The most frequent environmental pathogens of human concern are Salmonella typhi, Staphylococcus aureus, Listeria monocytogenes, vibrio cholera, Streptococcus pyogenes, Bacillus cereus, Klebsiella pneumonia, Enterobacter and Proteus species [1].

According to the $[2,3]$, the bacteria are large group of unicellular microorganisms, causing the most common fatal bacterial diseases such as the respiratory diseases. The streptococci are the main causative agent of the respiratory infections. They cause severe and invasive infections that have a mortality rate of $25 \%$ [4] including mild superficial skin infections to life-threatening systemic diseases such as tonsillitis / Strep throat, scarlet fever, impetigo, cellulitis, necrotizing asciitis, myositis and toxic shock syndrome, acute rheumatic fever and acute glomerulonephritis and also pneumonia caused by Streptococcus pneumoniae [5-8]. 
Pathogenic Streptococci have their specific virulent factors e.g. Streptococcus pneumoniae, which secretes pneumolysin that help them to act on the respiratory system of various age groups particularly of children that may be lethal to cause death [9]. Streptococcus pneumoniae is a major cause of various types of pneumonia e.g. $80 \%$ lobar pneumonia and $60 \%$ bronchopneumonia [1016].

Aim of the present study was to perform epidemiological and clinical study of Streptococcus pneumoniae between different age groups from Hyderabad region.

\section{Materials and Methods Sample collection}

Epidemiological survey of six months of the pneumonic patients and the hospitalized patients of various ages (3-65 years) was undertaken. The major part of the study was completed at Microbiology Research Laboratory (MRL), University of Jamshoro and minor part of work has been done at PCSIR (Pakistan Council Scientific and Industrial of Research) Laboratories Complex Karachi. About 374 samples of sputa and throat swabs were collected from various hospitals and clinical laboratories of Hyderabad and Jamshoro.

\section{Sampling method}

Sputa samples were collected in wide mouthed sterile bottles by allowing patients to cough and expectorate whereas the throat swabs were collected by allowing the patients to lay down their heads and the sterile commercial swab was rolled on the inflamed regions of throat after depressing the tongue with sterile depressor. The swabs were transferred to MRL in transport medium composed of brain heart infusion (BHI) broth supplemented with $1 \%$ glucose.

\section{Microbiological investigation}

All samples were investigated separately after collection on the specific days and time. Morphological examination by Gram staining technique was done and simultaneously culturing was also performed on blood agar, chocolate agar, brain heart infusion agar, china blue lactose agar, china blue maintol agar, mannitol salt agar, sulfide indole motility medium, nutrient gelatin and triple sugar iron agar. Sugar reactions were determined by inoculating culture in various sugar tubes. Test cultures were also applied for the determination of coagulase, catalase and oxidase production [17, 18]. Antibiotic discs sensitivity test of 21 antibiotics was performed on Mueller Hinton agar and blood agar plates by lawning the test culture uniformly by sterile swab to observe sensitivity test of the isolated culture after incubation at $37^{\circ} \mathrm{C}$ for 24 hours [17].

\section{Calculations and statistical analysis}

All analytical determinations were performed at least in triplicate and reported as mean $(n=2 \times 3)$.

\section{Results and Discussion}

The work was carried out to determine the ratio of infection in three age groups including children of 3-16, adults of 17-45 and old aged persons of 45-65 years. The results of investigation revealed that $S$. pneumoniae is gram-positive diplococci, having kidney shaped morphology which is examined by gram staining technique.

Pure culture study of 261 specimens (including 97 samples of sputa of adults and old age group, 164 throat swabs from children) were undertaken. It was observed that $37.33 \%$ sputa and $17.71 \%$ throat swabs were positive showing $S$. pneumoniae as pathogen. The isolates produced alpha hemolytic colonies on blood agar plate after 48 hours of incubation and reduced surface growth on China blue mannitol agar (Fig. 1 plate-A \& B). In-vitro growth on various other media is given in Table 1 . Sulfide indole motility medium is used to assess the ability of the culture for motility, indole reaction and hydrogen sulphide production by $S$. pneumoniae which show negative reaction because they are non motile and can not produce tryptophanase enzyme as well as does not reduce sodium thiosulphate in the medium (Table 1). 


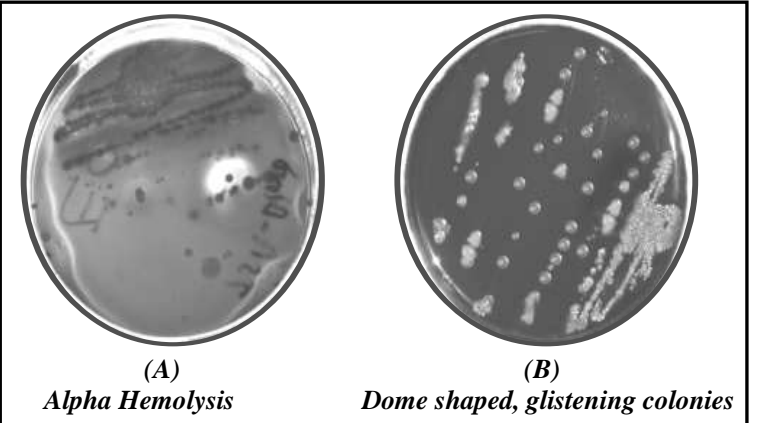

Growth of Streptococcus penumoniae on blood agar (A) and Chocolate agar (B) after 48 hours of incubation.

Figure 1. Hemolytic colonies of Streptococcus pneumoniae and sugar fermentation reactions

Table1. Cultural characters of Streptococcus pneumoniae on various growth media.

\begin{tabular}{|c|c|}
\hline Media & Cultural characters \\
\hline $\begin{array}{l}\text { Nutrient } \\
\text { agar }\end{array}$ & $\begin{array}{l}\text { Smooth, convex, rounded colonies with entire } \\
\text { margin }\end{array}$ \\
\hline $\begin{array}{l}\text { Brain Heart } \\
\text { Infusion } \\
\text { agar }\end{array}$ & Small, rounded, convex glistening, pale colonies \\
\hline Blood agar & $\begin{array}{l}\text { Rounded, convex, small to medium, grayish } \\
\text { colonies with no hemolytic zone after } 24 \text { hours but } \\
\text { dome shaped, rounded medium to large, scattered } \\
\text { colonies with zone of alpha hemolysis after } 48 \\
\text { hours. }\end{array}$ \\
\hline $\begin{array}{l}\text { China blue } \\
\text { manitol agar }\end{array}$ & $\begin{array}{l}\text { Surface growth, small pin pointed colonies with } \\
\text { irregular margins }\end{array}$ \\
\hline $\begin{array}{l}\text { China Blue } \\
\text { lactose agar }\end{array}$ & Reduced surface growth, no visible colony \\
\hline $\begin{array}{l}\text { Tryptic Soy } \\
\text { agar }\end{array}$ & $\begin{array}{l}\text { Rounded, smooth, convex, grayish white to pale } \\
\text { colonies }\end{array}$ \\
\hline BHI broth & $\begin{array}{l}\text { Uniform turbidity and whitish deposits after } 48 \\
\text { hours }\end{array}$ \\
\hline
\end{tabular}

Biochemical reactions revealed that the $S$. pneumoniae is gram positive, non acid fast, coagulase negative, oxidase negative and gelatin positive. They are capable to ferment sugars including glucose, lactose, maltose, galactose, fructose, sucrose, starch, and raffinose due to the specific enzymes produced by test species during sugar fermentation (Table 2).

Antimicrobial activity to the pneumococci shows resistance to the cefspan, septran, cravit, pipemetic acid, azomax, bacitracin, and penicillin. However pneumococci are sensitive to clithromycin, optochin, cefizox, genatamycin, minocyclin, levoflaxacin, and vancomycin. There were intermittent zones of inhibition to the claforan, nalidixic acid, amoxycillin, fosfomycin, fortum, and erythromycin (Table 3).

Table 2. Biochemical and sugar fermentation reaction of Streptococcus pneumoniae.

\begin{tabular}{lc}
\hline Coagulase & Biochemical Reactions \\
Catalase & Negative \\
Oxidase & Negative \\
Gel hydrolysis & Negative \\
\hline SIM agar & Positive \\
& Non motile \\
& Indole Negative \\
& Sodium sulfide reduction Negative \\
\hline Glucose, Galactose, Fructose, Maltose, Lactose, Sucrose, Starch, \\
Raffinose gives positive results.
\end{tabular}

Table 3. Various antibiotics used for determination of antibioticsensitivity of Streptococcus pneumoniae on Mueller Hinton agar.

\begin{tabular}{lc}
\multicolumn{1}{c}{ Antibiotics used } & Zone \\
\hline Cefspan & Resistant \\
Septran & \\
Cravit & \\
Pipemetic acid & \\
Azomax & \\
Bacitracin & \\
Penicillin & \\
\hline Clithromycin & \\
Cefizox & \\
Clafran & Sensitive \\
Amoxycillin & \\
Fosfomycin & \\
Minocin & \\
Gentamycin & \\
Optochin & \\
Levoflaxicin & \\
Vancomycin & \\
\hline Nalidixic acid & \\
Fortum & \\
Erythromycin & \\
\hline & \\
\hline
\end{tabular}

Current investigation revealed that environmental conditions of Hyderabad and Jamshoro had high incidence of microbial pollution. This study shows the prevalence of 
pneumonia $27.53 \%$. Highest ratio of the pathogen (S. pneumonia) was reported from throat swabs of old age people and children however little percentage in sputa of children and old age group was determined (Fig. 2). This might be due to the defective immune response against the pathogen or due to frequent use of un-prescribed antibiotics [19].
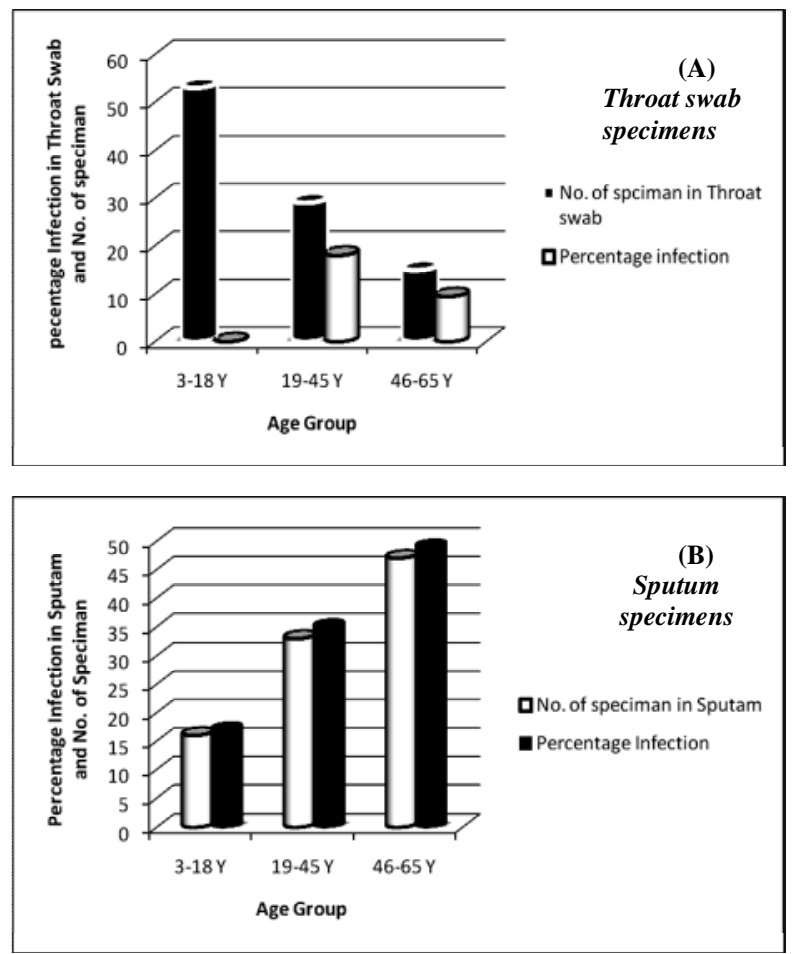

Figure 2. A \& B shows the various specimens collected from different hospitals and laboratories of various age group with percentage of prevalence of pneumonia in Hyderabad and Jamshoro.

Alpha hemolysis on blood agar was due to the virulent factor produced by test organism. Brain heart infusion agar promotes the growth of organism which requires high protein from calf brain and beef heart infusion and amino acid source for energy and growth. Reduction of growth on China blue mannitol agar may be due to the inhibitory effect of basic dye methylene blue present in a medium [20]. Gel hydrolysis was due to the production of proteolytic enzyme gelatinase which degrade gelatin and liquefies the medium into water. Antibiotic susceptibility test showed sensitivity of isolated pathogen to clithromycin, optochin, cefizox, genatamycin, minocyclin, levoflaxacin, and vancomycin, which was reported to be the effective treatment of pneumonia in various health centers of Hyderabad and Jamshoro. A few antibiotics showed intermediate zone and no zone, which may be due to the error in sample collection and resistance to the antibiotics respectively [21].

\section{Conclusion}

The streptococcal infections have been occurring in various areas of the Hyderabad and Jamshoro, which revealed that $S$. pneumoniae is one of the life threatening microorganisms with a greater ratio of infection in the children and old age persons but rarely in adults. It is concluded that, due to carelessness, improper diagnosis and mismanagement of chemotherapy, pneumonia may be the main cause among the children. It is also concluded that, antibiotics have more or less effects on the growth of test organisms but the antibiotic including clithromycin, fosfomycin, minocin, clafran, cefizex, fortum, gentamycin, amoxicillin and optochin showed the highest zone of inhibition as compared to the other effective antibiotics on growth of the test organism $S$. pneumoniae.

\section{References}

1. R. M. Anderson and M. M. Robert. Infectious diseases of humans, dynamics and control, Oxford University press, (1992), New York

2. J. K. Fredrickson, J. M. Zachara, D. L. Balkwill, D. Kennedy, S.W. Li, H.

M.Kostandarithes, M. J. Daly, M. F. Romine and F. J. Brockman. Appl. Environ. Microbiol., 70 (2004) 4230. doi: 10.1128/AEM.70.7.4230-4241.2004

3. C. L. Sears. Anaerobe., 11 (2005) 247. doi: 10.1016/j.anaerobe.2005.05.001)

4. C. O. K. Woese and M. Wheelis. Proc. Natl. Acad. Sci. US., 87 (1990) 4576. doi: $10.1073 /$ pnas.87.12.4576

5. R. Gupta. Crit. Rev. Microbiol., 26 (2000) 111.doi: 10.1080/10408410091154219

6. R. F. Doolittle. Curr. Opin. Struct. Biol., 15 (2005) 248. doi: 10.1016/j.sbi.2005.04.001

7. T. Cavalier-Smith. Int. J. Syst. Evol. Microbiol., 52 (2002) 7. doi: 10.1099/00207713-52-1-7 
8. M. P. Weinstein, M. L. Towns,S. M. Quartey, S. Mirrett, L. G. Reimer, G. Parmigiani, and L. B. Reller. Clin. Lab. Med., 14 (1994) 9.doi: 10.1093/clind/24.4.584

9. H. L. Chopra. Text Book Medical Microbiology, ( $1^{\text {st }}$ edn). Seema Pub New Delhi, (1985) 258-287.

10. J. P. Duguid, B. P. Marmion and R. H. A. Swam. Mackie and McCartney Medical Microbiology. ( $1^{\text {st }}$ edn.) Churchill Livingstone, (1978) 257.

11. S. B. Suillia and Shanthram. General Microbiology, (2 ${ }^{\text {nd }}$ edn). Oxford and IBH Pub. New Delhi, (2008) 501.

12. P. V. R. Raman. Essentials of Microbiology, (1 ${ }^{\text {st }}$ edn). CSB Pub. NY, (2005) 127.

13. C. A. Mims, J. H. L. Playfair, I. M. Roitt, D. Wakelin and R. Williams. Medical Microbiology, ( $3^{\text {rd }}$ edn). Mosby Pub. UK, (1993) 224.

14. R. Bhatia and R. L. Ichhpujani. Essential of Medical Microbiology, (1 ${ }^{\text {st }}$ edn). Jaypee Brothers Medical Pub. New Delhi. (1994) 244.
15. A. M. Khan. Basic Medical Microbiology. ( $1^{\text {st }}$ edn). Published by Time Publisher, (2001) 156, 169.

16. D. R. Arora. Textbook of Microbiology. $\left(2^{\text {nd }}\right.$ edn). CBS Pub. New Delhi, (2006) 227.

17. M. Cheesbrough. District Laboratory Practice in Tropical Countries, $\left(2^{\text {nd }}\right.$ edn $)$. Cambridge University, UK, (2000) 434.

18. T. R. Johnson, S. and C. L. Case. Laboratory Experiments in Microbiology, Benjamin/ Cummings Publishing Co. Inc. UK, (2013) 414.

19. E. Heise. Diseases associated with immunosuppression. Environ. Health Perspect, 43 (1982) 9. https://www.ncbi.nlm.nih.gov/pmc/articles/P MC1568899/

20. A. M. Khan and N. Mahmood. Laboratory Manual of Microbiology, ( $5^{\text {th }}$ edn). Time Publisher, (1999) 3-144.

21. P. Hastings, S. Rosenberg and A. Slack. Trends Microbiol., 12(2004) 401. doi: 10.1016/j.tim.2004.07.003 